\title{
Density-matrix renormalization-group studies of the spin-half Heisenberg system with dimerization and frustration
}

\author{
R. Chitra*, Swapan Pati**, H. R. Krishnamurthy*,+, \\ Diptiman Sen ${ }^{\dagger,+}$ and S. Ramasesha**,+ \\ * Physics Department, Indian Institute of Science, \\ Bangalore 560012, India \\ ** Solid State and Structural Chemistry Unit, \\ Indian Institute of Science, Bangalore 560012, India \\ $\dagger$ Centre for Theoretical Studies, Indian Institute of Science, \\ Bangalore 560012, India \\ + Jawaharlal Nehru Centre for Advanced Scientific Research, \\ Indian Institute of Science, Bangalore 560012, India
}

\begin{abstract}
Using the density matrix renormalization group technique, we study the ground state phase diagram and other low-energy properties of an isotropic antiferromagnetic spin-half chain with both dimerization and frustration, i.e., an alternation $\delta$ of the nearest neighbor exchanges and a next-nearest-neighbor exchange $J_{2}$. For $\delta=0$, the system is gapless for $J_{2}<J_{2 c}$ and has a gap for $J_{2}>J_{2 c}$ where $J_{2 c}$ is about 0.241. For $J_{2}=J_{2 c}$, the gap above the ground state grows as $\delta$ to the power $0.667 \pm 0.001$. In the $J_{2}-\delta$ plane, there is a disorder line $2 J_{2}+\delta=1$. To the left of this line, the peak in the static structure factor $S(q)$ is at $q_{\max }=\pi$ (Neel phase), while to the right of the line, $q_{\max }$ decreases from $\pi$ to $\pi / 2$ as $J_{2}$ is increased to large values (spiral phase). For $\delta=1$, the system is equivalent to two coupled chains as on a ladder and it is gapped for all values of the interchain coupling.
\end{abstract}

PACS numbers: 75.10.Jm, 75.50.Ee 


\section{INTRODUCTION}

One of the most widely studied spin models is the isotropic spin-half Heisenberg antiferromagnetic chain. This model is Bethe ansatz soluble ${ }^{1,2}$ for uniform nearestneighbor (NN) exchange constants. Since we can map the spin-half model in one dimension to a system of interacting spinless fermions by the Jordan-Wigner transformation, we expect a Peierls type of dimerization in this system when it is coupled to phonons. The dimerized model, with exchange constants alternating as $1 \pm \delta$, is not exactly solvable, although many numerical and approximate analytic results exist for this model. There has also been considerable interest in the spin-half chain ${ }^{3}$ with both $\mathrm{NN}$ and next-nearest-neighbor $(\mathrm{NNN})$ antiferromagnetic interactions $J_{2}$. Interestingly, the ground state of this model can be solved exactly ${ }^{4}$ for $J_{2}=0.5$. The ground state is doubly degenerate for the ring and the two states are representable as the two Kekule structures of the valence-bond theory.

The heuristic phase diagram (Fig. 1) of the ground state for a model that incorporates the effects of both dimerization and frustration, i.e., the $J_{2}-\delta$ model is known, although there has been no systematic study of this model in the $J_{2}-\delta$ plane. The ground state is exactly known for points on the line $2 J_{2}+\delta=1^{5}$. Analytical and numerical studies ${ }^{6-8}$ of the model on the $\delta=0$ line show a transition from a gapless phase for $J_{2}<J_{2 c}$ to a gapped phase for $J_{2}>J_{2 c}$. The value of $J_{2 c}$ has been accurately computed to be $0.2411 \pm 0.0001$ in Ref. 8. The $J_{2}=0, \delta>0$ line corresponds to a dimerized spin chain. Another interesting line on the phase diagram is $\delta=1$ which corresponds (cf. Fig. 2) to coupled spin chains with $J_{2}$ as the intrachain and 2 as the interchain coupling constants. Little else is known about the general $J_{2}-\delta$ model. Numerical studies of this model have been largely confined to exact low-lying states of small systems $N \leq 22$ on the $\delta=0$ line.

In this paper we present a systematic study of the various regions of this phase 
diagram using the density matrix renormalization group (DMRG) method ${ }^{9}$. Using this technique we have studied both open chains and rings of sizes ranging between 200 and 300 sites, depending upon the parameters of the model. In section II, we give a brief introduction to the method and present some computational details. In section III, we present our results and discuss them.

\section{THE HAMILTONIAN AND THE DMRG TECHNIQUE}

The Hamiltonian for the spin-half chain we have studied is given by

$$
\begin{aligned}
H & =H_{0}+H_{1}+H_{2} \\
H_{0} & =\sum_{i=1}^{2 N-1} \vec{S}_{i} \cdot \vec{S}_{i+1} \\
H_{1} & =-\delta \sum_{i=1}^{2 N-1}(-1)^{i} \vec{S}_{i} \cdot \vec{S}_{i+1} \\
H_{2} & =J_{2} \sum_{i=1}^{2 N-2} \vec{S}_{i} \cdot \vec{S}_{i+2},
\end{aligned}
$$

where $J_{2}$ is the NNN exchange and $\delta$ is the NN exchange alternation parameter. (Note that we have set the average $\mathrm{NN}$ exchange $J_{1}=1$ ). In the open chain with an even number of sites, the dimerization is chosen to make the exchange constant for the terminal bonds strong $(1+\delta)$. The topology of the chain being studied is shown in Fig. 2.

The DMRG technique involves systematically building up the chain to a desired number of sites starting from a very short chain by adding two sites at a time. The initial chain of $2 n$ sites, with $n$ a small enough integer, is diagonalized exactly and the reduced density matrix for the left $n$ sites is computed from the ground state of the $2 n$ chain Hamiltonian by integrating over the states of the right $n$ sites. The density matrix is diagonalized and a matrix representation of the $n$-site Hamiltonian is obtained in a truncated basis using $m$ basis vectors which are the eigenvectors of the 
density matrix corresponding to its $m$ largest eigenvalues. The Hamiltonian matrix for the $2 n+2$ chain is then obtained in the $(2 s+1)^{2} m^{2}$ dimensional direct product subspace obtained using the truncated basis of the left and the right parts of the $2 n$ chain and the full space of the two additional spins which are inserted in the middle. After obtaining the ground state of the $2 n+2$ chain in the truncated basis, the density matrix of half the chain, now with $n+1$ sites, is obtained. The procedure is repeated to obtain the ground state of the $2 n+4$ chain. The iteration is stopped on reaching the desired chain length.

The DMRG technique works best for spin-half chains with short-range interactions. The accuracy depends crucially on the number of eigenvalues of the density matrix, $m$, that are retained. For spin-half chains with NN interactions only, $m=32$ gives results that approximate very well to the infinite system results. In systems with longer range interactions, it becomes necessary to keep track of the spin matrices in the most recent basis, corresponding to all the sites which interact with the new spins that are introduced at a given DMRG step. While the DMRG algorithm is devised to minimize the errors due to truncation of the Hamiltonian matrix, the algorithm does not necessarily retain the spin matrices very accurately. The limited success of the DMRG technique for two-dimensional lattices can be attributed to this fact, since a two-dimensional lattice is topologically equivalent to a one-dimensional lattice with very long-range interactions. In the case of spin-half chains with $\mathrm{NN}$ and NNN interactions, the spin matrices that appear in the Hamiltonian at any stage would have undergone at most two transformations, and the results we find for $\mathrm{m}=64$ compare well with exact numerical diagonalizations of chains upto 22 sites.

\section{A FIELD THEORY FOR THE NEEL PHASE}

Before presenting our numerical results, we briefly discuss a field theory for the low-energy and long-wavelength modes of the spin chain. In the Neel phase, the low- 
energy physics is well described by an $O(3)$ nonlinear sigma model (NLSM) with a topological term ${ }^{10,12}$. (The field variable is an unit vector). This led Haldane to predict that integer and half-integer spin chains will have qualitatively different behaviour for $\delta=0$, in that the former have a gap in their excitation spectrum while the latter do not. This prediction has received support from numerical calculations and experiments.

Since our primary interest in this paper lies in the spin-half chain, it is convenient to use a different approach which is specific to these chains. This is the technique of bosonization discussed by Luther and Peschel $^{11}$ and Affleck ${ }^{12}$. In this approach, we first carry out a Jordan-Wigner transformation on the spin chain to obtain a model of interacting spinless fermions on a lattice

$$
\begin{aligned}
S_{i}^{z} & =\psi_{i}^{\dagger} \psi_{i}-\frac{1}{2} \\
S_{i}^{+} & =\psi_{i} \exp \left[i \pi \sum_{j<i} \psi_{j}^{\dagger} \psi_{j}\right] .
\end{aligned}
$$

The spin-up and spin-down states at a site correspond respectively to states with and without a fermion. The continuum limit of the transformed Hamiltonian can be bosonized to obtain a tractable bosonic theory in $1+1$ dimensions. For our system, this just produces the theory of a free 'relativistic' massless boson. The corresponding Lagrangian density is given by

$$
\mathcal{L}=\frac{1}{2}\left(\partial_{\mu} \phi\right)^{2},
$$

where $\phi$ is a bosonic field. The scaling dimension of an operator $\exp (i \alpha \phi)$ is therefore $\alpha^{2} / 4 \pi$. The relation between a spin operator and the continuum field $\phi$ is given by

$$
S^{z}(x) \sim(-1)^{x / a} \sin (\sqrt{2 \pi} \phi),
$$

where $a$ is the underlying lattice constant. Since the spin Hamiltonian is isotropic, it is sufficient to consider the $S^{z} S^{z}$ correlations. A straightforward evaluation of this correlation function yields

$$
\left\langle S^{z}(x) S^{z}(0)\right\rangle \sim \frac{(-1)^{x / a}}{|x|} .
$$


This power-law correlation implies the absence of a gap above the ground state. Eq. (5) generally has corrections like $\log (x / a)$ because the field theory has a marginal operator $\mathcal{O}$. In the spin chain, this corresponds to the NNN exchange $J_{2}$.

There is a crtical value $J_{2 c}$ such that $\mathcal{O}$ is marginally irrelevant for $J_{2}<J_{2 c}$ and marginally relevant for $J_{2}>J_{2 c}$. At $J_{2 c}$, the field theory is exactly conformally invariant and there are no logarithmic corrections. The spectrum at that point is described by a specific conformal field theory. In particular, the gap between the ground state and the low-lying excited states scales with the chain length as $1 / N$. Further, the first excited states with $S=0$ and $S=1$ are exactly degenerate. For $J_{2}<J_{2 c}$, the excited $S=0$ state has a higher energy than the $S=1$ states. For $J_{2}>J_{2 c}$, the $S=0$ state becomes degenerate with the ground state as $N \rightarrow \infty$, and the triplet states are separated from the two ground states by a finite gap. One can therefore determine $J_{2 c}$ accurately as the point where the singlet and triplet excited states are degenerate for large values of $N^{7,8}$.

We can treat the spin-Peierls term $H_{1}$ in Eq. (1) as a perturbation on $H_{0}$ for small values of $\delta$. Using the above procedure, we obtain the bosonic representation of $H_{1}$ in the continuum limit ${ }^{12}$ as

$$
H_{1} \sim \delta \cos (\sqrt{2 \pi} \phi) .
$$

This is a relevant operator with scaling dimension $\frac{1}{2}$ and it therefore produces a gap

$$
\Delta \sim \delta^{2 / 3} .
$$

Similarly, the change in the ground state energy caused by the perturbation in (6) scales as

$$
E_{o}(\delta)-E_{o}(0) \sim \delta^{4 / 3} .
$$

These results are perturbative ${ }^{14}$ and they are valid only for small $\delta$. Further, there are generally corrections of order $\log (\delta)$ in Eqs. (7) and (8) because of the presence of 
the marginal operator. However if we are exactly at the critical point $J_{2 c}$, there are no logarithmic corrections to (7) and (8).

For $J_{2}>J_{2 c}$ and $\delta=0$, there is a gap between the two degenerate ground states (both $S=0$ ) and the first excited state $(S=1)$. Near $J_{2 c}$, the gap $\Delta$ has an essential singularity of the form

$$
\Delta \sim \exp \left(-\frac{A}{J_{2}-J_{2 c}}\right)
$$

Hence the gap is numerically indistinguishable from zero unless $J_{2}$ is greater than about 0.3 .

Before ending this section, we note that a different field theory is required in the spiral phase (which, for $\delta=0$, sets in beyond $J_{2}=0.5$ for spin-half). This field theory is a NLSM based on a $S O(3)$ matrix unlike the unit vector field in the NLSM for the Neel phase. The main feature of this new field theory is that there is no topological term and therefore no qualitative difference between integer and half-integer spin chains ${ }^{13}$. There should be gap above the ground state(s) in either case.

\section{RESULTS AND DISCUSSION}

We now present the phase diagram of the model and the various results obtained by us using DMRG. We also compare these with the existing analytical and numerical results. This is done in four subsections: A. The frustrated model with $\delta=0 ; \mathrm{B}$. The dimerized model with $J_{2}=$ constant; C. The general $J_{2}-\delta$ model; and, D. Coupled spin chains with $\delta=1$.

\section{A. The frustrated spin chain $\left(J_{2}>0, \delta=0\right)$}

Very few exact results are known for this model. The ground state is exactly solvable for $J_{2}=0.5$. For cyclic boundary conditions, the ground state is doubly degenerate with the wave functions being given by the two possible Kekule structures, 
namely, the two patterns of singlets which can be formed by neighboring sites.

$$
\begin{aligned}
& \psi_{1}=[1,2][3,4] \ldots[2 N-1,2 N] \\
& \psi_{2}=[2 N, 1][2,3] \ldots[2 N-2,2 N-1],
\end{aligned}
$$

where $[i, j]$ denotes the normalized singlet combination of the spins on sites $i$ and $j$.

The field theory studies by Haldane on the frustrated model revealed the existence of a transition from a gapless phase for $J_{2}<J_{2 c}$ to a gapped phase for $J_{2}>J_{2 c}$. He estimated $J_{2 c}$ to be 0.16 . A direct computation of the gap obtained by exact diagonalization of small systems ${ }^{3}$ placed $J_{2 c}$ near 0.30 . However this turns out to be an unreliable method because of the essential singularity in Eq. (9) as $J_{2}$ approaches $J_{2 c}$ from above. The most reliable method of computing $J_{2 c}$ is the one based on the crossing of the excited singlet and triplet states extrapolated to infinite system size through finite size scaling. Ref. 8 obtained a value of $J_{2 c}=0.2411 \pm 0.0001$ by this method. In the next subsection, we use this value of $J_{2 c}$ to study the relation between the gap and $\delta$.

We have confirmed that the ground state is doubly degenerate for $J_{2}>J_{2 c}$, as required by the Lieb-Schultz-Mattis theorem for gapped spin-half systems. Fig. 3 summarizes our results for the extrapolated gap for $0<J_{2} \leq 1$. The gap increases for $J_{2}>J_{2 c}$ as a function of $J_{2}$ until about $J_{2}=0.7$ beyond which it decreases. In the limit of very large $J_{2}$, the gap should once again vanish since the system then consists of two decoupled nearest neighbor chains. We find that this behaviour sets in even at a relatively small value of $J_{2}=0.8$. Fig. 4 shows the convergence of the gap with increasing chain length in the gapped $\left(1 / N^{2}\right)$ as well as in the gapless $(1 / N)$ regions, and illustrates the validity of our extrapolation procedures.

\section{B. Dimerized model $\left(J_{2}=\right.$ constant,$\left.\delta>0\right)$}

At $J_{2 c}$, we have calculated the gap $\Delta$ for various values of $\delta$ on spin chains of upto 300 sites with the open boundary condition. The ground state is a singlet and 
the lowest excitation is a triplet. The triplet is therefore obtained as the lowest energy state in the $S^{z}=1$ sector within the DMRG formalism. The minimum chain length at which the infinite chain behaviour can be expected to set in scales as the correlation length and hence depends inversely on $\Delta$. For $\delta=0.007$ (which is the smallest $\delta$ we have studied), the infinite chain behaviour is expected for lengths greater than about 200 sites.

Fig. 5 shows that the $\log -\log$ plot of the gap versus $\delta$ is linear. The exponent for the gap is given by the slope of this straight line which is $0.667 \pm 0.001$. Previous numerical studies using exact diagonalization of spin chains with upto 22 sites had placed the value of this exponent between 0.9 and $1.0^{15,16}$, while those using finite size scaling placed it closer to $0.75^{17}$. However these studies were all at $J_{2}=0$, hence they were subject to errors due to the logarithmic corrections. Fig. 6 shows the $\log -\log$ plot of the change in the ground state energy $E_{o}(\delta)-E_{o}(0)$ versus $\delta$. The slope of the straight line is $1.251 \pm 0.001$ which is somewhat different from the field theory result of $4 / 3$.

For a 'relativistic' massive theory which differs from a massless theory by a small perturbation (by small we mean that the 'velocity of light' is not changed), the mass gap $\Delta$ and the correlation length $\xi$ should be inversely related to each other. In Fig. 7 , we plot the product $\xi \Delta$ versus $\delta$ for $J_{2}=0$, and find that this does appear to be the case.

\section{The general $J_{2}-\delta$ model}

The ground state of the model is known to be exactly solvable along the line $2 J_{2}+\delta=1$. The presence of the dimerization $\delta$ lifts the degeneracy of the two ground states at $J_{2}=0.5$. One of the Kekule states continues to be the ground state while the other is no longer an eigenstate of the Hamiltonian. Choosing the state $\psi_{1}$ in Eq. (9) 
as a trial wave function, we obtain a variational energy per site equal to

$$
E_{0}=-\frac{3}{8}(1+\delta)
$$

Using the Rayleigh-Ritz variational principle, it is then easy to show that $E_{0}$ saturates the lower bound to $\langle H\rangle$ provided $^{5} 2 J_{2}+\delta=1$. Fig. 8 shows the variation of the gap along this line.

The general model exhibits a gap in the excitation spectrum for all points $\left(J_{2}, \delta\right)$ not covered by the special cases discussed above. The ground state energies for various values of $J_{2}$ and $\delta$ are given in Table 1 .

To characterize the ground state further, we computed the equal time spin-spin correlation function at many points in the phase diagram. The system sizes chosen for these calculations were varied with $J_{2}$ and $\delta$ so as to reasonably approximate the infinite chain behaviour. We then computed the static structure factor $S(q)$ defined as the Fourier transform of the correlation function. Some information about the type of long-range order (LRO), if any, can be obtained from the dependence of $S(q)$ on $q$. The classical limit $(S \rightarrow \infty)$ predicts Neel order for $J_{2}<0.25$ and a (coplanar) spiral order for $J_{2}>0.25$. In other words, $S(q)$ has a peak at $q_{\max }=\pi$ for $J_{2}<0.25$ and $q_{\max }=\cos ^{-1}\left(-1 / 4 J_{2}\right)$ for $J_{2}>0.25$. The periodicity of the ground state is $2 \pi / q_{\max }$.

The quantum model has no LRO but exhibits a short-range order characterized by some $q_{\max }$ (and a finite correlation length if there is a gap in the spectrum). Earlier numerical studies ${ }^{3}$ of the $S=1 / 2$ system revealed marked deviations from the large- $S$ (classical) results. They showed that $q_{\max }=\pi$ for $J_{2} \leq 0.5$ and $q_{\max }<\pi$ for $J_{2}>0.5$, with $q_{\max }$ approaching $\pi / 2$ for large $J_{2}$.

We have calculated $S(q)$ for rings of sizes 100 and 150 at the points indicated in Fig. 9. The structure factor peaks at $q_{\max }=\pi$ for all points along the $J_{2}=0$ line. On the $\delta=0$ line, $S(q)$ peaks at $q_{\max }=\pi$ for $J_{2} \leq 0.5$ and $q_{\max }<\pi$ for $J_{2}>0.5$ with $q_{\max }$ approaching $\pi / 2$ for very large $J_{2}$ as shown in Fig. 10 . In the full $J_{2}-\delta$ 
plane, $S(q)$ shows a very interesting change of behaviour across the line $2 J_{2}+\delta=1$. For points to the left of the line, $S(q)$ peaks at $q_{\max }=\pi$ while for points to the right of the line, $S(q)$ peaks at $q_{\max }<\pi$. However, for points close to the line on the right hand side, $S(q)$ shows a very broad distribution near $q=\pi$ and we cannot identify a clear maximum in the plot (Fig. 11).

The correlation length $\xi$ is a minimum along the entire line $2 J_{2}+\delta=1$, reflecting a highly disordered ground state. The main features in the $S(q)$ plots seem to be in conformity with this behavior of $\xi$. We can therefore call $2 J_{2}+\delta=1$ a disorder line. The disorder line separates the ground states with $q_{\max }=\pi$ and $q_{\max }<\pi$ as shown in Fig. 9.

\section{Coupled spin chains $(\delta=1)$}

For $\delta=1.0$ and $J_{2}>0$, the model corresponds to two coupled spin chains as discussed earlier. The phase diagram of coupled spin-half chains was studied in Ref. 18. We have examined the dependence of the gap on the scaled strength of the interchain coupling $J_{i} \equiv 2 / J_{2}$. This was calculated for a $2 \times 100$ ladder. In Fig. 12, we see a gap in the spectrum even for very small values of $J_{i}$ as shown. Our results for the excitation gaps are in agreement with the studies of Barnes et al ${ }^{19}$ which suggested the presence of a non-zero energy gap between the singlet ground state and the triplet excited states for all antiferromagnetic interchain couplings.

The convergence of the ground state energy per site for this system is shown in Fig. 13. The fluctuation in the ground state energy with the total number of sites is because of the alternation between even and odd number of sites on a single chain. The amplitude of the fluctuation is large for weak interchain couplings and gets damped as the coupling between the chains increases. The energy per site when the intrachain and interchain exchange constants are equal is 0.5780 . This value is intermediate between the ground state energy per site for the isotropic Heisenberg anitferromagnet on the 
two-dimensional square lattice $(-0.67)$ and the ground state energy per site on a chain $(-0.4431)$. The advantage of our method is that it is still treated as a single chain problem and thus avoids the pit falls of DMRG in two dimensions. The accuracy of the method for the ladder is the same as that for the single chain.

\section{SUMMARY}

To conclude, we have studied the NN and NNN antiferromagnetic spin-half Heisenberg chain with a dimerization, $\delta$, in the NN exchange. When $\delta$ is zero, there is a critical value $J_{2 c}=0.2411$ below which the system is gapless and above which the system is gapped. For the dimerized chain with the critical value of the NNN interaction, the energy gap above the ground state scales as $\delta$ to the power $0.667 \pm 0.001$, while the change in the ground state energy $E_{o}(\delta)-E_{o}$ scales with the power $1.251 \pm 0.001$. In the $J_{2}-\delta$ plane, we find a disorder line $2 J_{2}+\delta=1$ to the left of which the static structure factor $S(q)$ peaks at $q_{\max }=\pi$. To the right of this line, $q_{\max }$ gradually decreases from $\pi$ to $\pi / 2$ for large $J_{2}$. For $\delta=1$, the model corresponds to coupled chains as on a ladder. The system is gapped for all values of antiferromagnetic $J_{i}$. The energy per site is -0.57804 for $J_{i}=1.0$ which lies in between the values for the one-dimensional and two-dimensional (square lattice) NN antiferromagnetic systems.

\section{REFERENCES}

1. J. des Cloizeaux and J.J. Pearson, Phys. Rev. 128, 2131 (1962) and references therein.

2. J.C. Bonner and M.E. Fisher, Phys. Rev. 135, A640 (1964).

3. T. Tonegawa and I. Harada, J. Phys. Soc. Jpn. 56, 2153 (1987). 
4. C. K. Majumdar and D. K. Ghosh, J. Math. Phys. 10, 1388 (1969); C. K. Majumdar, J. Phys. C 3, 911 (1970).

5. B. S. Shastry and B. Sutherland, Phys. Rev. Lett. 47, 964 (1981).

6. F. D. M. Haldane, Phys. Rev. B 25, 4925 (1982); R. Jullien and F. D. M. Haldane, Bull. Am. Phys. Soc. 28, 344 (1983)

7. I. Affleck, D. Gepner, H. J. Schulz and T. Ziman, J. Phys. A 22, 511 (1989).

8. K. Okamoto and K. Nomura, Phys. Lett. A 169, 433 (1992).

9. S. R. White, Phys. Rev. Lett. 69, 2863 (1992); Phys. Rev. B 48, 10345 (1993).

10. F. D. M. Haldane, Phys. Lett. 93A, 464 (1983); Phys. Rev. Lett. 501153 (1983).

11. A. Luther and I. Peschel, Phys. Rev. B 12, 3908 (1975).

12. I. Affleck, in Fields, Strings and Critical Phenomena, eds. E. Brezin and J. ZinnJustin (North-Holland, Amsterdam, 1989); I. Affleck and F.D.M. Haldane, Phys. Rev. B 36, 5291 (1897).

13. S. Rao and D. Sen, Nucl. Phys. B 424, 547 (1994); D. Allen and D. Senechal, to appear in Phys. Rev. B (1995).

14. M. C. Cross and D. S. Fisher, Phys. Rev. B 19, 402 (1979).

15. S. Inagaki and H. Fukuyama, J. Phys. Soc. Jpn. 52, 2504 (1983); S. Ramasesha and Z. G. Soos, Solid State Commun. 46, 509 (1983).

16. Z. G. Soos, S. Kuwajima, J.E. Mihalick, Phys. Rev. B 32, 3124 (1985).

17. J. C. Bonner and H.W.J. Blote, Phys. Rev. B 25, 6959 (1982).

18. S. P. Strong and A. J. Millis, Phys. Rev. Lett. 69, 2419 (1992).

19. T. Barnes, E. Dagotto, J. Riera and E. S. Swanson, Phys. Rev. B 47, 3196 (1993). 


\section{Table Captions}

1. Ground state energy per site $E_{o}$ for various values of $J_{2}$ and $\delta$.

2. Ground state energy per site for coupled spin chains for various values of $J_{i}$. 


\begin{tabular}{|c|c|c|}
\hline$J_{2}$ & $\delta$ & $E_{o}$ \\
\hline $\begin{array}{l}0.2411 \\
0.2411 \\
0.2411 \\
0.2411 \\
0.2411 \\
0.2411 \\
0.2411 \\
0.2411 \\
0.2411 \\
0.2411 \\
0.2411\end{array}$ & $\begin{array}{l}0.000 \\
0.007 \\
0.014 \\
0.020 \\
0.028 \\
0.040 \\
0.057 \\
0.080 \\
0.160 \\
0.230 \\
0.320\end{array}$ & $\begin{array}{l}-0.401866 \\
-0.402681 \\
-0.403766 \\
-0.404844 \\
-0.406430 \\
-0.409051 \\
-0.413132 \\
-0.419154 \\
-0.442862 \\
-0.465728 \\
-0.496844\end{array}$ \\
\hline $\begin{array}{l}0.10 \\
0.20 \\
0.25 \\
0.30 \\
0.40 \\
0.50 \\
0.60 \\
0.70 \\
0.80 \\
1.00 \\
\end{array}$ & $\begin{array}{l}0.0 \\
0.0 \\
0.0 \\
0.0 \\
0.0 \\
0.0 \\
0.0 \\
0.0 \\
0.0 \\
0.0 \\
\end{array}$ & $\begin{array}{l}-0.42517 \\
-0.40885 \\
-0.40045 \\
-0.39284 \\
-0.38028 \\
-0.37500 \\
-0.38079 \\
-0.39711 \\
-0.42138 \\
-0.48565 \\
\end{array}$ \\
\hline $\begin{array}{l}0.45 \\
0.48 \\
0.55 \\
0.15 \\
0.25 \\
0.40 \\
0.15 \\
0.20 \\
0.30 \\
0.48\end{array}$ & $\begin{array}{l}0.07 \\
0.10 \\
0.10 \\
0.20 \\
0.35 \\
0.50 \\
0.60 \\
0.80 \\
0.80 \\
0.80\end{array}$ & $\begin{array}{l}-0.40130 \\
-0.41281 \\
-0.41610 \\
-0.46329 \\
-0.50727 \\
-0.56611 \\
-0.60033 \\
-0.67613 \\
-0.67966 \\
-0.69256\end{array}$ \\
\hline
\end{tabular}

Table 1 


\begin{tabular}{|c|c|}
\hline$J_{i}$ & $E_{o}$ \\
\hline 0.020 & -0.44320 \\
0.040 & -0.44337 \\
0.066 & -0.44434 \\
0.133 & -0.44320 \\
0.200 & -0.44741 \\
1.000 & -0.57804 \\
\hline
\end{tabular}

Table 2 


\section{Figure Captions}

1. Heuristic phase diagram for the spin-half chain. The solid line along $\delta=0$ from $J_{2}=0$ to $J_{2 c}$ is gapless; the rest of the diagram is gapped. The line $2 J_{2}+\delta=1$ separates the Neel phase from the spiral phase.

2. Schematic picture of the antiferromagnetic exchanges in the chain.

3. Dependence of the gap $\Delta$ on $J_{2}$ for $\delta=0$.

4. Convergence of the singlet-triplet gap $\Delta$ with $1 / N$ for $J_{2}=0.5$ (gapped system) and $J_{2}=0.2$ (gapless) at $\delta=0$.

5. Log-log plot of the gap $\Delta$ versus $\delta$ for $J_{2}=0.2411$.

6. Log-log plot of the change in the ground state energy $E_{o}(\delta)-E_{o}(0)$ versus $\delta$ for $J_{2}=0.2411$.

7. Plot of the product of the correlation length $\xi$ and the gap $\Delta$ versus $\delta$ for $J_{2}=0$.

8. Gap $\Delta$ versus $\delta$ along the line $2 J_{2}+\delta=1$.

9. Behaviour of the structure factor $S(q)$ in the $J_{2}-\delta$ plane.

10. Plot of $q_{\max }$ (in degrees) versus $J_{2}$ for $\delta=0$.

11. $S(q)$ versus $q$ (in degrees) for various values of $J_{2}$ and $\delta$.

12. Gap versus the interchain coupling $J_{i}$ for coupled chains.

13. Convergence of the ground state energy per spin $E_{o}$ with system size for coupled chains with $J_{i}=0.04$. 